\title{
Development of a recurrent pleural effusion in a patient with pulmonary arterial hypertension treated with imatinib
}

\author{
Olga M. Fortenko ${ }^{1}$, Lana Melendres-Groves ${ }^{2}$, Alice Richter ${ }^{1}$, Xiaobo Liao ${ }^{1}$, \\ Edda Spiekerkoetter ${ }^{1}$, Roham Zamanian ${ }^{1}$, Vinicio A. De Jesus Perez ${ }^{1^{*}}$ \\ ${ }^{1}$ Stanford University Medical Center, Stanford, USA; *Corresponding Author: vdejesus@stanford.edu \\ ${ }^{2}$ University of New Mexico, Albuquerque, USA
}

Received 9 October 2012; revised 16 November 2012; accepted 25 November 2012

\begin{abstract}
Pulmonary arterial hypertension (PAH) is a devastating disease associated with progressive elevation in pulmonary pressures that eventually leads to chronic right heart failure and death. At present, agents with vasodilatory properties are being used to palliate the symptoms associated with PAH but there is a need for therapies that can prevent or even reverse established disease. Several lines of evidence have suggested that tyrosine kinase inhibitors like imatinib may have a role in reducing progression and improving outcomes in these patients, but their side effect profile is unclear. We present a case of a 55-year-old female with PAH secondary to connective tissue disease treated with triple PAH specific therapy and compassionate-use imatinib who developed a massive right pleural effusion. Despite multiple therapeutic thoracentesis and aggressive diuresis, the pleural effusion continued to re-accumulate necessitating chest tube placement. Resolution of the pleural effusion was finally achieved after imatinib was held, arguing that the patient's presentation likely was a drug-related event. We believe that our case highlights a serious adverse reaction to imatinib therapy and stresses the need for more studies to evaluate the safety profile of this medication in patients with PAH.
\end{abstract}

Keywords: Pulmonary Hypertension; Imatinib; Tyrosine Kinase Inhibitors; Pleural Effusion

\section{INTRODUCTION}

Pulmonary arterial hypertension (PAH) is characterized by structural changes in the pulmonary vasculature leading to increased pulmonary artery pressure and pulmonary vascular resistance (PVR), which ultimately progress to right ventricular failure and death [1]. The pathologic changes in the pulmonary arteries consist of smooth muscle proliferation, intimal fibrosis and characteristic plexiform lesions that lead to narrowing and obstruction of pulmonary vasculature [2]. Despite the currently available pulmonary vasodilator therapies, prognosis remains dismal. Platelet-derived growth factor (PDGF) has recently been identified as a therapeutic target in PAH. PDGF is involved in signal transduction pathways associated with smooth muscle proliferation and is overexpressed in the pulmonary arteries of $\mathrm{PAH}$ patients [3]. Imatinib, a tyrosine kinase inhibitor that is currently FDA approved for the treatment of malignancies such as chronic myeloid leukemia (CML) and gastrointestinal stromal tumors, has been shown to improve the clinical status of patients as well as reverse pulmonary vascular remodeling in animal models of PAH $[3,4]$. Furthermore, results from phase 2 and ongoing phase 3 clinical trials suggest that imatinib may improve the functional status and hemodynamics in patients with severe PAH [2,5]. However, despite its therapeutic promise, the safety profile of imatinib in PAH patients has not been fully characterized. This is relevant to the future therapeutic utility of these agents, as serious adverse reactions with imatinib have been reported in cancer patients and could have devastating consequences in PAH patients.

We report a case of a recurrent pleural effusion in a patient with World Health Organization (WHO) Group I pulmonary arterial hypertension associated with connective tissue disease that developed after receiving imatinib therapy for several months, and stress the need for caution when using these agents outside their FDA approved applications.

\section{CASE REPORT}

A 55 year-old white female with a 7-year history of 
pulmonary arterial hypertension associated with limited scleroderma presented to the Stanford Pulmonary Hypertension $(\mathrm{PH})$ clinic complaining of worsening shortness of breath and fatigue. She was initially diagnosed with PAH in 2005 at an outside hospital and treated with inhaled iloprost for several years. Her care was transferred to Stanford in 2009, at which time a right heart catheterization showed a mean pulmonary arterial pressure of $35 \mathrm{mmHg}$. She presented with worsening symptoms and evidence of right ventricular failure in 2010 and was initiated on intravenous epoprostenol. Over time, her medical regimen was expanded to include ambrisentan and tadalafil. Despite triple therapy, she developed worsening right ventricular failure and renal dysfunction, leading to frequent hospital admissions for intravenous diuretics. Given her end-stage disease and poor candidacy for lung transplant due to her obesity, she was initiated on experimental therapy with imatinib in October 2011. Two months later, she came to the Stanford PH clinic complaining of dyspnea on exertion, 3-pillow orthopnea and abdominal distention, which prompted the team to admit her for management of decompensated right heart failure.

In addition to her PAH and limited scleroderma, the patient's past medical history was also significant for type 2 diabetes mellitus, obstructive sleep apnea treated with CPAP, morbid obesity (BMI $39.6 \mathrm{~kg} / \mathrm{m}^{2}$ ), and hypothyroidism. Family history was significant for coronary artery disease in her father and multiple sclerosis in her mother. Patient was divorced and had a healthy, adult son. She was a former smoker with a thirty pack-year tobacco history but had quit in July 2009. She admitted to cocaine and methamphetamine use in her early twenties but had been sober for thirty years. She was formerly employed as a medical assistant but had been on disability for the last several years. Medications at the time of admission included epoprostenol infusion at 54 $\mathrm{ng} / \mathrm{kg} / \mathrm{min}$, tadalafil $40 \mathrm{mg}$ PO daily, ambrisentan $10 \mathrm{mg}$ PO daily, imatinib $200 \mathrm{mg}$ PO daily, furosemide $100 \mathrm{mg}$ po BID, spironolactone $100 \mathrm{mg}$ PO daily, bumetanide 1 mg PO daily, hydroxychloroquine $400 \mathrm{mg}$ PO daily, omeprazole $40 \mathrm{mg}$ PO daily, aspirin $325 \mathrm{mg}$ PO daily, rosuvastatin $10 \mathrm{mg}$ po QHS, levothyroxine $88 \mathrm{mcg}$ PO daily, sertraline $100 \mathrm{mg}$ PO daily, amitriptyline $25 \mathrm{mg}$ PO daily, and hydrocodone-acetaminophen 7.5 - $750 \mathrm{mg}$ PO TID as needed for pain. She reported an allergy to sildenafil, which caused a diffuse rash.

Physical examination revealed an afebrile, morbidly obese female with a blood pressure of 106/57, heart rate of 110 beats per minute, respiratory rate of 20, oxygen saturation of $87 \%$ on $4 \mathrm{~L}$ via nasal cannula. Her weight was $101.6 \mathrm{~kg}$, which was stable compared to one week ago. She appeared to be in moderate respiratory distress, speaking in incomplete sentences due to breathlessness. Examination of her neck revealed jugular venous distention up to her mandible. Cardiac examination was significant for tachycardia and an accentuated second heart sound, but no appreciable murmur, rubs or gallops. Respiratory exam revealed tachypnea with use of accessory muscles of respiration. Breath sounds were diminished on the right middle and lower lung fields with inspiratory rales and dullness to percussion. No egophany was noted. Lower extremity pitting edema was present and was $1+$ bilaterally. The remainder of her physical examination was unremarkable.

Laboratory work up was significant for an NT-proBNP of $2037 \mathrm{pg} / \mathrm{mL}$, which was close to patient's baseline. A chest X-ray obtained in the emergency department showed a large right pleural effusion, which was freely movable on lateral decubitous films. A diagnostic and therapeutic thoracentesis was performed with 1.5 liters of serous fluid removed. The pleural fluid met Light's criteria for an exudative effusion (total protein ratio 0.7). Cell count showed $72 \%$ neutrophils, $24 \%$ lymphocytes, and 3\% monocytes. Microbiologic studies of the pleural fluid were negative for an infectious etiology, and cytology was negative for malignancy. A transthoracic echocardiogram (TTE) revealed a moderate right ventricular enlargement with moderately reduced right ventricular systolic function, a right ventricular systolic pressure (RVSP) of $62 \mathrm{mmHg}$ with a right atrial pressure of $20 \mathrm{mmHg}$, and normal left ventricular size and systolic function. Overall, there was no significant change compared to a TTE obtained 1 month prior to admission.

Despite aggressive therapy with dobutamine and furosemide infusions, patient's right pleural effusion reaccumulated, requiring a repeat therapeutic thoracentesis and ultimately a pig-tail catheter placement. A CT scan of her chest did not show any underlying pulmonary pathology to account for the effusion. Per our rheumatology colleagues, there was no evidence to suggest a flare of CTD on physical exam or laboratory studies. Patient had lost $10 \mathrm{lbs}$ since admission with the diuretic regimen. However, the right pleural effusion re-accumulated when the pigtail catheter was clamped for 48 hours. At this point, we decided to explore the possibility of a drug induced pleuritis and began to review her medication list for possible culprits. Given the known potential for imatinib to induce volume overload and prior reports describing pleural and pericardial effusion occurring in patients using other tyrosine kinase inhibitors (see discussion), we decided to hold imatinib and assess subsequent pleural fluid reaccumulation. Three days after discontinuing imatinib, the patient's respiratory status remained stable and no further fluid accumulation was observed prompt- 
ing the removal of the chest tube.

\section{DISCUSSION}

Imatinib is a tyrosine kinase inhibitor that targets BcrAbl kinase and has been approved for the treatment of CML and several other malignancies [6]. Research has shown that imatinib also targets PDGF receptors, which are involved in pulmonary vascular smooth muscle proliferation prompting speculation regarding a potential therapeutic role for this agent in PAH [3].

Ghofrani et al. recently showed that imatinib decreased PVR and improved cardiac output in patients with severe WHO group $1 \mathrm{PAH}$ in a Phase II study that included 56 patients with $\mathrm{PAH}$ [2]. In this study, imatinib appeared to be well tolerated in most patients, with the most common reported side effects being nausea, muscle cramps and rashes. However, imatinib-associated fluid retention has been described in several reports, including a longterm, multicenter trial of patients with CML that documented fluid retention in $60 \%$ of patients treated with imatinib [6]. Interestingly, there are few case reports in the literature of imatinib-associated pleural effusions in patients with CML, most of these appearing to be associated with higher doses of the drug [7]. Serosal inflammation occurs more commonly with dasatinib, a secondline tyrosine kinase inhibitor for CML. The incidence of pleural effusions with dasatinib has been reported to range from $7 \%$ to $35 \%$, compared to less than $1 \%$ with imatinib [8]. The pathogenesis is unclear but is thought to be related to immune deregulation and has been shown to be steroid-responsive [9].

To date, the Food and Drug Administration (FDA) has not approved imatinib for use in PAH. An extension of the IMPRES trial reported peripheral edema occurring in $16.8 \%$ of patients [10]. Other serious adverse events, including cerebral hemorrhage, were also reported. The FDA cancelled its advisory committee meeting scheduled for September 2012 intended to discuss the approval of imatinib as an adjunctive therapy for $\mathrm{PAH}$, requesting more information [11]. Finally, it is worth noting that a recently published study has raised concerns regarding the possible association of dasatinib with development of PAH in patients receiving this therapy for CML [12]. Whether this represents an effect of this particular drug or a class effect is unclear at this time but it strongly argues that caution should be used when testing this agents in patients who already carry the burden of this disease.

\section{CONCLUSION}

We report a case of a recurrent pleural effusion occurring in a patient with $\mathrm{PAH}$ treated with imatinib for 3 months. After left ventricular failure, infection, malignnancy and scleroderma-associated serositis were excluded as alternative causes of the pleural fluid, imatinib was discontinued with complete resolution of the effusion. This case highlights the importance of evaluating for potential medication side effects and the need for further studies to elucidate the safety profile of imatinib in the treatment of PAH.

\section{ACKNOWLEDGEMENTS}

We thank the patient and her family for all the help provided and for their permission to use their clinical history to illustrate this case.

\section{REFERENCES}

[1] Handoko, M.L., de Man, F.S., Allaart, C.P., Paulus, W.J., Westerhof, N. and Vonk-Noordegraaf, A. (2010) Perspectives on novel therapeutic strategies for right heart failure in pulmonary arterial hypertension: Lessons from the left heart. European Respiratory Review, 19, 72-82. doi:10.1183/09059180.00007109

[2] Ghofrani, H.A., Morrell, N.W., Hoeper, M.M., et al. (2010) Imatinib in pulmonary arterial hypertension patients with inadequate response to established therapy. American Journal of Respiratory and Critical Care Medicine, 182, 1171-1177. doi:10.1164/rccm.201001-01230C

[3] Perros, F., Montani, D., Dorfmuller, P., et al. (2008) Platelet-derived growth factor expression and function in idiopathic pulmonary arterial hypertension. American Journal of Respiratory and Critical Care Medicine, 178, 81-88. doi:10.1164/rccm.200707-1037OC

[4] Schermuly, R.T., Dony, E., Ghofrani, H.A., et al. (2005) Reversal of experimental pulmonary hypertension by PDGF inhibition. The Journal of Clinical Investigation, 115, 2811-2821. doi:10.1172/JCI24838

[5] Hoeper, M.M., et al. (2011) Imatinib in pulmonary arterial hypertension, a randomized, efficacy study (IMPRES). Data presented at the European Respiratory Society (ERS) Annual Congress. Abstract No. 413.

[6] Druker, B.J., Guilhot, F., O’Brien, S.O., et al. (2006) Five-year follow-up of patients receiving imatinib for chronic myeloid leukemia. The New England Journal of Medicine, 355, 2408-2417.

[7] Gerogianni, I., Kouloumenta, V., Zigoulis, P., et al. (2006) A 50-year-old man with pleural effusion and chronic myelogenous leukemia. The Internet Journal of Pulmonary Medicine, 6, 115. doi:10.5580/115

[8] Kelly, K., Swords, R., Mahalingam, D., Padmanabhan, S. and Giles, F.J. (2009) Serosal inflammation (pleural and pericardial effusions) related to tyrosine kinase inhibitors. Targeted Oncology, 4, 99-105.

[9] Bergeron, A., Rea, D., Levy, V., et al. (2007) Lung abnormalities after desatinib treatment for chronic myeloid leukemia. American Journal of Respiratory and Critical Care Medicine, 176, 814-818. 


\section{doi:10.1164/rccm.200705-715CR}

[10] Hoeper, M.M., Barst, R.J., Chang, H.J., et al. (2012) Imatinib safety and efficacy: Interim analysis of IMPRES extension study in patients with pulmonary arterial hypertension. American Journal of Respiratory and Critical Care Medicine, 185, A2495.

[11] Young, D. (2012) Scrip intelligence.
http://www.scripintelligence.com/home/Novartis-yanks-i matinib-PAH-NDA-FDA-wants-more-data-cancels-AdCo $\underline{\mathrm{m}-334095}$

[12] Montani, D., Bergot, E., Gunther, S., et al. (2012) Pulmonary arterial hypertension in patient treated by dasatinib. Circulation, 125, 2128-2137. 